\title{
The Scutellaria Flavone, Oroxylin A, Improves Attention-Deficit/ Hyperactivity Disorder Related Behaviors in Spontaneously Hypertensive Rats
}

\author{
Seo Young Yoon 5 , Mi Sook Chun 5 , Yong Soo Lee ${ }^{1}$, Hae II Park ${ }^{2}$, Chan Young Shin ${ }^{3}$, \\ Jong Hoon $\mathrm{RYu}^{4}$, and Jae Hoon CheONG* \\ Uimyung Institute for Neuroscience, Sahmyook University, Seoul 139-742, Republic of Korea \\ ${ }^{1}$ Department of Pharmacology College of Pharmacy, Duksung Women's University, Seoul 132-714, Republic of Korea \\ ${ }^{2}$ College of Pharmacy, Kangwon National University, Chunchon 200-701, Republic of Korea \\ ${ }^{3}$ Department of Pharmacology College of Medicine, Konkuk University, Seoul 143-701, Republic of Korea \\ ${ }^{4}$ Department of Oriental Pharmaceutical Science College of Pharmacy, KyungHee University, Seoul 130-701, Republic of Korea \\ ${ }^{5}$ Department of Pharmacy, Sahmyook University, 26-21, Kongreung 2-dong, Nowon-goo Seoul139-742, Republic of Korea
}

(Received October 8, 2008; Revised November 18, 2008; Accepted November 21, 2008)

\begin{abstract}
Oroxylin A is a flavonoid isolated from Scutellaria baicalensis, which is one of the most important medicinal herbs in traditional Korean medicine. In this study, we investigated the psychopharmacological activities of oroxylin A using the open field, rota-rod, balanced wire and plus-maze tests in Spontaneously Hypertensive Rats (SHR) and Wistar Kyoto Rats (WKY). Oroxylin A reduced hyperactivity in SHR (ADHD animal model) although it tended to increase locomotor activity in WKY. Methylphenidate did not reduce hyperactivity. Oroxylin A alleviated impulsive behaviors such as rearing, the percentage of moving time to the central area and the tendency to move into an unstable condition (open area in elevated plus-maze). Methylphenidate also reduced the percentage of staying time in the central area and the tendency to move into an unstable condition. Both oroxylin $A$ and methylphenidate enhanced motor attention in SHR and WKY. Oroxylin A antagonized the muscimol $\left(G A B A_{A}\right.$ receptor agonist)-induced Clcurrent and its action was similar to that of bicuculline $\left(G A B A_{A}\right.$ receptor antagonist). The effects of oroxylin $A$ may be caused by the antagonism at the $G_{A B A}$ receptor. Thus, oroxylin A may be a candidate of drug for treatment of ADHD.
\end{abstract}

Keywords: Scutellaria flavone, Behavior, Oroxylin A, ADHD, GABA

\section{INTRODUCTION}

Attention deficit hyperactivity disorder (ADHD) is the most prevalent childhood developmental disorder affecting between $3 \%$ and $7 \%$ of school-age children. ADHD is characterized by persistent hyperactivity, impulsivity and inattention that are differentially expressed in three subtypes: primarily inattentive, primarily hyperactive/impulsive or combined in type (Fone and Nutt, 2005). The aetiology of $A D H D$ is unclear. Although the neurobiological basis of ADHD is unresolved, recent advances in molecular genetics and brain imaging have improved our

\footnotetext{
${ }^{*}$ Corresponding author

Tel: +82-2-3399-1605, Fax: +82-3399-1617

E-mail: cheongjh@syu.ac.k

${ }^{5}$ These authors contributed equally to this work.
}

understanding of $A D H D$, and increasing indirect evidence implicates dopaminergic hypofunction in the frontal lobes and basal ganglia. Recent single positron emission tomography analysis suggests that the striatal dopamine transporter (DAT) levels are markedly increased in patients with ADHD (Krause et al., 2003). There is, however, considerable debate as to whether the increased DAT levels reported in imaging studies cause a reduction in synaptic DA or whether elevated DA triggers the rise in DAT levels (Solanto, 2002). Spontaneously hypertensive rats (SHR) are the best characterized and also currently the most appropriate model of ADHD. SHR exhibit all the behavioral characteristics of ADHD: impaired sustained attention without obvious sensory problems, motor impulsiveness, and hyperactivity that is not present in novel, non-threatening situations but develops over time when reinforcers are infrequent (Sagvolden 
et al., 2005).

Studies on the roles of GABAergic dysfunction in ADHD are lesser in scope compared to studies on dopaminergic dysfunction although GABAergic dysfunction has been shown to directly or indirectly contribute to the pathogenesis of ADHD. The GABAergic and dopaminergic systems exhibit a high interconnectivity, with dopamine innervating GABAergic cell bodies, dendrites and axon terminals in either an excitatory or inhibitory manner (Brummelte et al., 2008)

The psychostimulants methylphenidate and amphetamine are the drugs of choice for ADHD, providing clinical benefits against the three core symptoms in $70-80 \%$ of patients (Elia et al., 1999). But, occasional marked side effects including loss of appetite, insomnia and, less commonly, motor tics and rebound symptoms following rapid drug withdrawal make the development of alternative medication desirable (Kollins et al., 2001). Potential therapies such as adrenergic receptor agonists, glutamatergic agents, gamma aminobutyric acid (GABA) receptor antagonists and nicotine receptor agonists are being explored as future pharmacotherapies for ADHD.

Scutellaria baicalensis is one of the most important medicinal herbs in traditional Korean medicine. The root of $S$. baicalensis is widely employed in traditional Korean prescriptions. Wogonin, baicailin, baicalein, and oroxylin $A$, which are the major chemical constituents of this herb, are flavone derivatives that contain a phenylbenzopyrone nucleus (Hui et al., 2002; Lin and Shieh, 1996). The flavonoids isolated from this herb exhibit moderate affinities to the benzodiazepine receptor (Dekermendjian et al., 1999). Behavioral studies have demonstrated that the water extract of Scutellaria baicalensis exerts potent anxiolysis in mice without sedative and myorelaxant effects (Jeong et al., 2004). Flavonoids from S. baicalensis may have pharmacologically and clinically important activities including anxiolysis, anti-convulsion, muscle-relaxation, and sedative effects because they bind to benzodiazepine or GABA receptors (Hui et al., 2000). The psychopharmacological properties of flavonoids may be also differ although several groups have reported anxiolytic effects of flavonoids (Hui et al., 2002; Paladini et al., 1999; Salgueiro et al., 1997).

Oroxylin A (5,7-dihydroxy-6-methoxyflavone) is a flavonoid isolated from the roots of $S$. baicalensis (Tomimori et al., 1982). In previous studies, oroxylin A ameliorated scopolamine- or A 325 -35-induced memory impairment and protected neurons from transient cerebral hypoperfusion-induced neuronal damage (Kim et al., 2006; Kim et al., 2007 ; Kim et al., 2008). Oroxylin A (5 $\mathrm{mg} / \mathrm{kg}$ ) also reversed cognitive impairments in passive avoidance and the Y-maze test and improved escape latencies in training trials and increased swimming times and distances within the target zone of the Morris water maze (Kim et al., 2007). In addition, previous reports have revealed that oroxylin $A$ has an anti-oxidative and anti-inflammatory activities. Therefore, oroxylin A may have beneficial effects on oxidative stress or inflammation-induced memory and learning impairments. We previously demonstrated that oroxylin A has an awakening activity in mice that is similar to that of methylphenidate, a typical drug used to treat ADHD. These results suggest that oroxylin A may be available to ameliorate symptoms of ADHD.

Screening of traditional medicines has contributed invaluably to drug development and discovery. We have recently been characterizing the psychopharmacological properties of flavonoids isolated from Scutellaria baicalensis and found that one of its constituents, Oroxylin A, has awakening and memory enhancing effects. This profile led us to explore its potential application in ADHD by evaluating its ameliorating effects on ADHD behaviors as hyperactivity, impulsiveness and inattention. The purpose of this study was to evaluate whether oroxylin $A$ could be used to treat ADHD.

\section{MATERIALS AND METHODS}

\section{Animals and materials}

Male Spontaneously Hypertensive rats (SHR) (4 weeks of age) and male Wistar Kyoto rats (WKY) (4 weeks of age) used in this study were purchased from Hanlim Experimental Animal Co. (Hwasung, Korea). All animals were maintained on a standard light-dark cycle, at ambient temperature $\left(23 \pm 2{ }^{\circ} \mathrm{C}\right)$ and humidity $(55 \pm 5 \%)$ with free access to chow pellets and water. All animals were acclimated to their home cages for at least 6 days before testing. Animals were divided into control group, oroxylin A-treated group and methylphenidate-treated group of normal rats (WKY) and ADHD rats (SHR). The experimental groups, consisting of 8-10 animals per each drug and dose, were chosen by means of a randomized schedule. All tests took place between 10:00 and 16:00 h. Animal treatment and maintenance were in accordance with the Principle of Laboratory Animal Care (NIH publication No. 85-23 revised 1985) and the Animal Care and Use Guidelines of Sahmyook University, Korea

Oroxylin A was synthesized in the School of Pharmacy of Kangwon National University. Methylphenidate was supplied by Hwanin Pharmaceutical Co. (Ansung, 
Korea). Diazepam was purchased from Samjin Phamaceutical Co., LTD (Seoul, Korea). Muscimol, bicuculline and other materials were purchased from Sigma-Aldrich Co. (St. Louis, MO, USA). Oroxylin A was injected intraperitoneally at dosages of 2 and $10 \mathrm{mg} / \mathrm{kg}$. Animals in the control group were injected intraperitoneally with an equivalent volume of saline. Diazepam (2 mg/kg) or methylphenidate $(3 \mathrm{mg} / \mathrm{kg})$ was injected intraperitoneally into rats of the positive control group.

\section{Behavioral apparatus}

The equipment was located in the animal room, allowing the observer to view and observe the animals using a computer outside the room. The behavioral changes of the animals were monitored automatically using a computerized EthoVision system (Noldus IT b.v., Netherlands). In the locomotor activity, Rota-rod and elevated plus-maze tests, the behavioral parameters were analyzed by automatic systems.

\section{Locomotor activity}

The apparatus consisted of 9 black plastic boxes $(47 \times 347 \mathrm{~cm})$, and the field was bordered by $42-\mathrm{cm}$-high side walls. The total distance moved, total movement time and turn angles were monitored for 20 minutes after administration (Kim et al., 2003; Noldus et al., 2001).

\section{Elevated plus-maze test}

The elevated plus-maze box and arms were made of plastic. The apparatus consisted of two open arms (50×10 $\mathrm{cm}$ ), at right angles, with two of the resulting four arms enclosed by high walls of $20 \mathrm{~cm}$. Each of the four arms had a delimited central area of $10 \times 10 \mathrm{~cm}$. The entire apparatus was placed $50 \mathrm{~cm}$ above the floor. Animals were placed in the central square and allowed to explore the maze freely for 5 minutes. The parameters measured were the times spent in open and closed areas (Kim et al., 2003; Noldus et al., 2001). The impulsive animals spent more time in the open arms than the normal animal.

\section{Rota-rod evaluation}

The rota-rod test was used to assess whether oroxylin A caused motor attention or gross motor impairment in the animals. Twenty-four hours before the experiment, all mice were habituated to running on the rota-rod at a speed of $60 \mathrm{rpm}$ until they could remain there for $60 \mathrm{~s}$ without falling. The latency to the first fall was recorded and falling frequency was also measured for 20 minutes using a stopwatch (Lee et al., 2006; Farkas et al., 2005).

\section{Intracellular $\mathrm{Cl}^{-}$measurement assay}

Relative changes in intracellular $\mathrm{Cl}^{-}$concentration $\left(\left[\mathrm{Cl}_{\mathrm{i}}\right]_{\mathrm{i}}\right)$ in IMR-32 human neuroblastoma cells were monitored using a $\mathrm{Cl}^{-}$-sensitive indicator, $\mathrm{N}$-(6-methoxyquinolyl) acetoetylester (MQAE), developed by Verkman et al. (1989), according to the method of West and Molly (1996). Briefly, cells were washed twice and re-suspended at a concentration of $4 \times 10^{5} \mathrm{cells} / \mathrm{ml}$ in Hank's solution. Cells were incubated with MQAE dye overnight at a final concentration of $5 \mathrm{mM}$ at room temperature to load the dye into the cells. Fluorescence (excitation wavelength set at $365 \mathrm{~nm}$ and emission wavelength at $450 \mathrm{~nm}$ ) was monitored in a well-stirred cuvette. Experiments were performed at room temperature to minimize fluorescent dye loss. Data are presented as relative fluorescence $F_{0} / F$, where $\mathrm{F}_{0}$ is the fluorescence without $\mathrm{Cl}^{-}$ions and $\mathrm{F}$ is the fluorescence as a function of time.

The $F_{0} / F$ values are directly proportional to $\left[\mathrm{Cl}^{-}\right]_{\text {i. }}$ All fluorescence values were corrected for background fluorescence, which was separately determined using a HEPES-buffered KSCN solution containing $5 \mu \mathrm{M}$ valinomycin to maximally quench the MQAE ion-selective signal (Shumaker et al., 1999). In separate experiments the $\mathrm{F}_{0}$ value was determined by bathing the cells in $\mathrm{Cl}^{-}$-free $\left(\mathrm{KNO}_{3}\right)$ solution containing $10 \mathrm{mM}$ tributyltin and $10 \mathrm{mM}$ nigericin.

\section{Statistical analysis}

Data are expressed as the mean \pm S.E.M. ANOVA was used to compare the scores among the groups for one variable. This was followed by post hoc comparisons using the Newman-Keuls test.

\section{RESULTS}

\section{Hyperactivity test by measuring movement}

Previous studies have demonstrated that SHR are more hyperactive than their WKY counterparts. In this study, the total moving time and distance were significantly different between WKY and SHR. In figure 1, SHR exhibited a more pronounced activity/hyperactivity (measured as the total distance moved and the duration of movement) than WKY. Oroxylin A administration (2 and $10 \mathrm{mg} / \mathrm{kg}$ ) significantly reduced hyperactivity in SHR $(p<0.01)$ but not in WKY. Meanwhile, methylphenidate, a typical drug for treatment of ADHD, did not reduce hyperactive behavior in SHR and significantly increased distance moved $(p<0.01)$ and movement duration $(p<0.05)$ in WKY. 

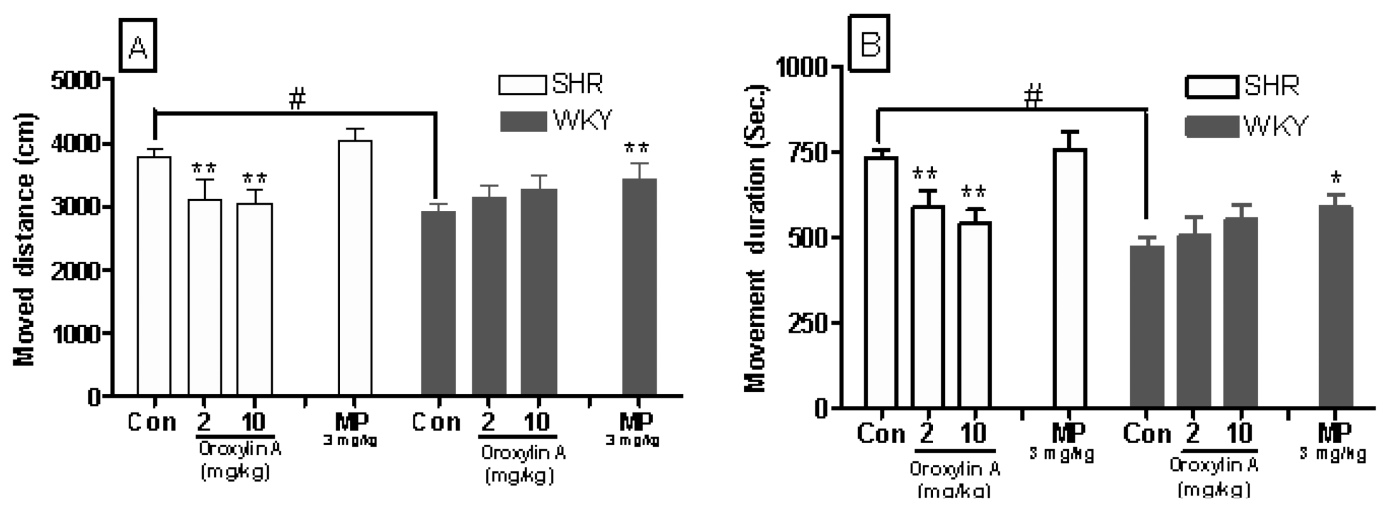

Fig. 1. Effect of oroxylin A on locomotor activity in SHR and WKY $(n=9)$. Each bar represents the mean \pm SEM of total distances moved $(A)$ and movement duration $(B)$ for 20 minutes. Control versus oroxylin $A$ or methylphenidate $(\mathrm{MPH}),{ }^{*} p<0.05{ }^{* *} p<0.01$; SHR control versus WKY control, \# $p<0.05$.

\section{Impulsiveness test by measuring rearing and behavior on the plus maze}

Impulsiveness was measured by observing the animals' rearing behavior, the tendency to go to the central area and activity on the plus maze. Animals showing impulsivity rear frequently and spend more time in the central area or the open arms rather than in the corners or closed areas. Figures 2 and 3 show that the rearing frequency and the percentage of time moving in the central area were considerably higher in SHR than in WKY. Oroxylin A treatment $(2$ and $10 \mathrm{mg} / \mathrm{kg}$ ) significantly reduced the rearing frequency $(p<0.01)$ in SHR but not in WKY. In addition, oroxylin A $(10 \mathrm{mg} / \mathrm{kg})$ significantly reduced the percentage of time moving in the central area in SHR but not in WKY. Methylphenidate also decreased the percentage of time moving in the central area in SHR but not in WKY. Methylphenidate failed to reduce rearing frequency in SHR but enhanced such behavior in WKY. As shown in figure 3, the time spent in the open or closed arms for 5 minutes differed significantly between strains. Oroxylin A (10 mg/kg) -treated SHR spent more time in the closed arms than salinetreated SHR. Methylphenidate also increased the percentage of staying time in closed arms in SHR.

\section{Motor attention test by measuring activity on the rota-rod}

The ability of the animal to stay on a rotating rod for long periods could represent sustained attention, a characteristic deficient in ADHD. As shown in figure 4, SHR stayed less time on the rota-rod than WKY. They also fell more frequently than WKY. Oroxylin A (10 mg/kg) and methylphenidate $(3 \mathrm{mg} / \mathrm{kg})$ significantly increased the animals' running time on the rota-rod and reduced their falling frequency. Therefore, both oroxylin A and methylphenidate enhanced motor attention in both strains.
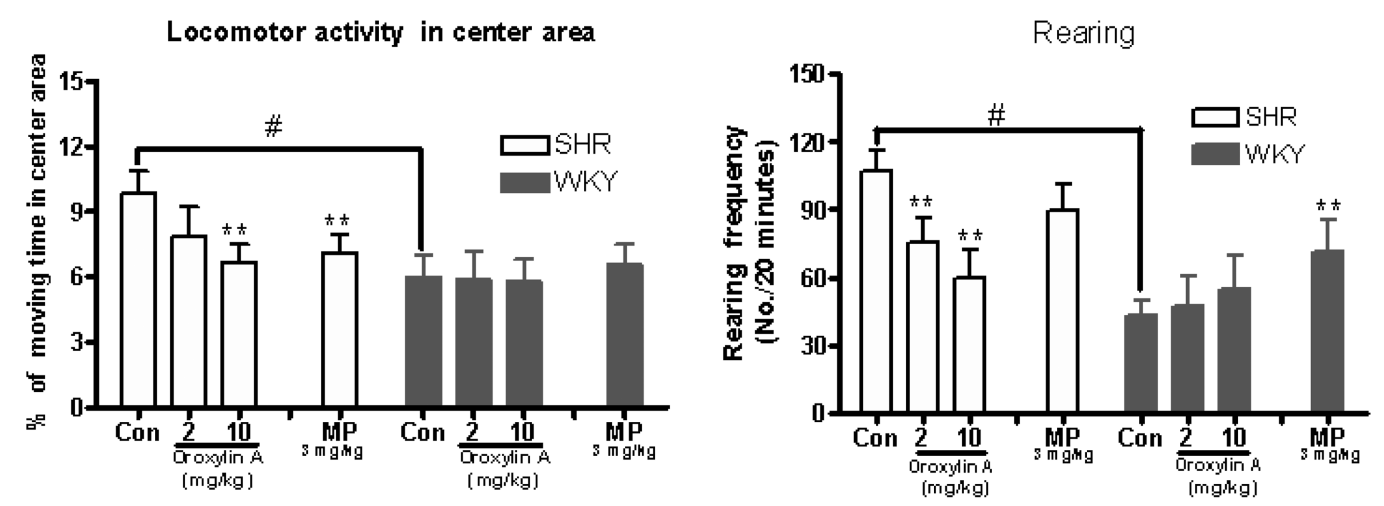

Fig. 2. Effect of oroxylin A on impulsivity in SHR and WKY $(n=9)$. Each bar represents the mean \pm SEM of \% of moving time in the central area (left) and rearing frequency (right) for 20 minutes. Control versus oroxylin A or methylphenidate $(\mathrm{MPH}),{ }^{*} \mathrm{p}<0.05{ }^{* *} \mathrm{p}<$ 0.01; SHR control versus WKY control, \# $\mathrm{p}<0.05$. 

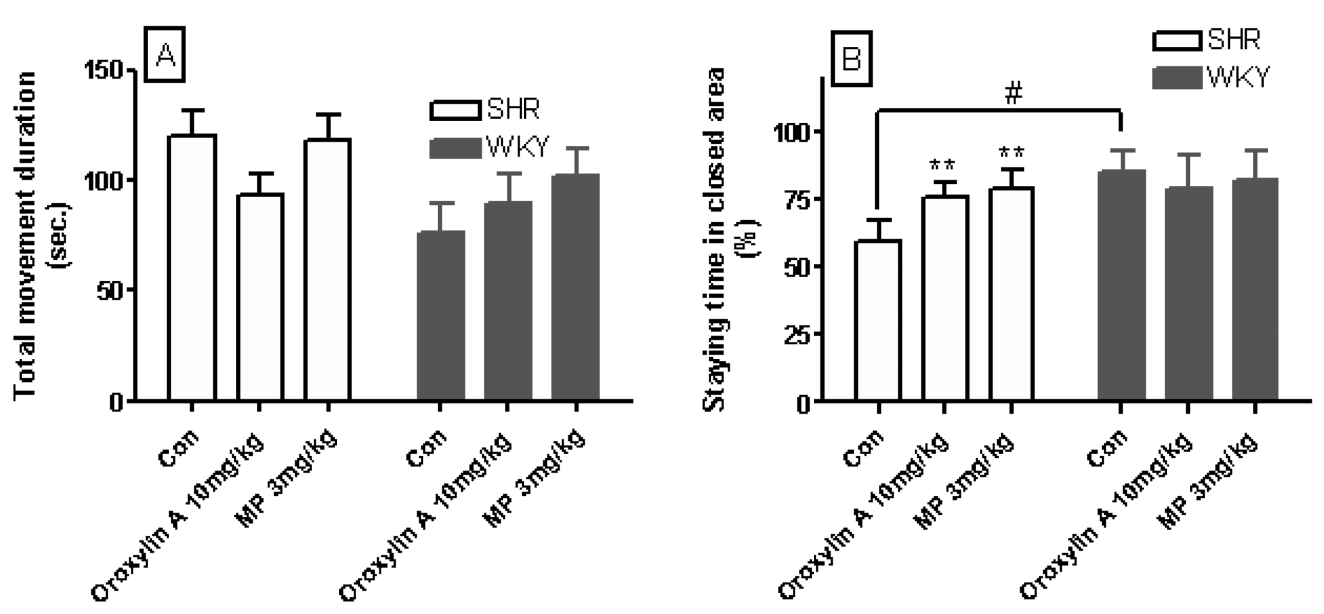

Fig. 3. Effect of oroxylin A on impulsivity in SHR and WKY $(n=9)$. Each bar represents the mean \pm SEM of movement duration in the elevated plus-maze (A) and \% of staying time in closed arms (B) for 5 minutes. Control versus oroxylin A or methylphenidate (MPH), ${ }^{*} p<0.05{ }^{* *} p<0.01$; SHR control versus WKY control, $\# p<0.05$.
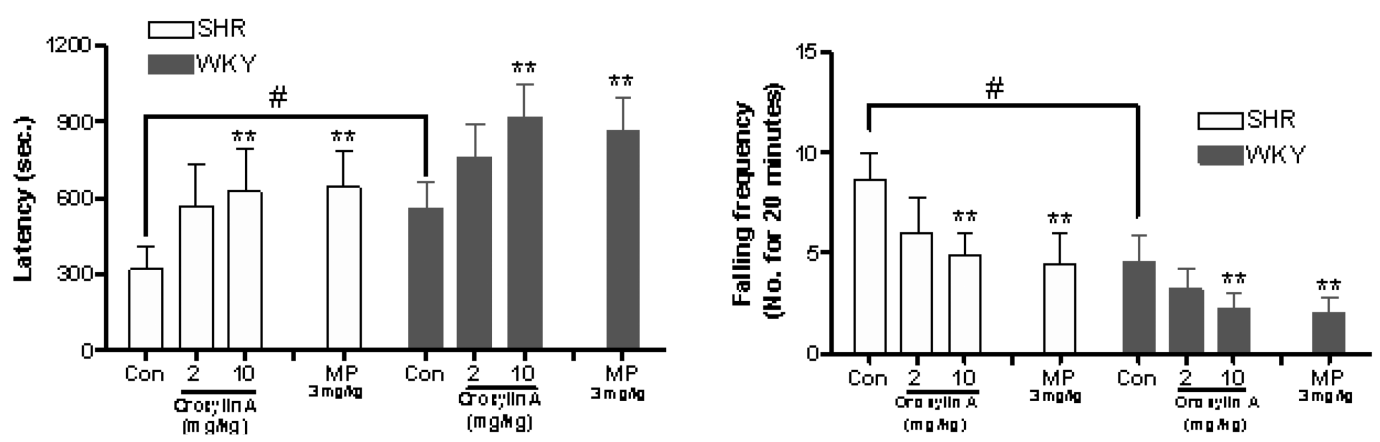

Fig. 4. Effect of oroxylin $A$ on motor attention on the rota-rod in SHR and WKY $(n=9)$. Each bar represents the mean \pm SEM of running times until fall from the rota-rod (left) and falling frequency for 20 minutes (right). Control versus oroxylin $A$ or methylphenidate (MPH), ${ }^{*} p<0.05{ }^{* *} p<0.01$; SHR control versus WKY control, \# $p<0.05$

\section{Influx of $\mathrm{Cl}^{-}$ion into cell}

Figure 5 shows the electrophysiological change. Treatment with muscimol, a GABA receptor agonist increased $\mathrm{Cl}^{-}$influx into the intracellular area. Conversely, oroxylin A inhibited the $\mathrm{Cl}^{-}$influx induced by muscimol treatment. Its effect was similar to that of bicuculline, a GABA receptor antagonist.

\section{DISCUSSION}

Behavioral studies have demonstrated that Scutellaria flavonoids exert potent anxiolysis in mice without sedation and myorelaxation (Jeong et al., 2004; Michael et al., 2003). In a previous study, we described the pharmacological characterization of active flavonoids from $S$. baicalensis, such as oroxylin $A$, baicalein and wogonin.

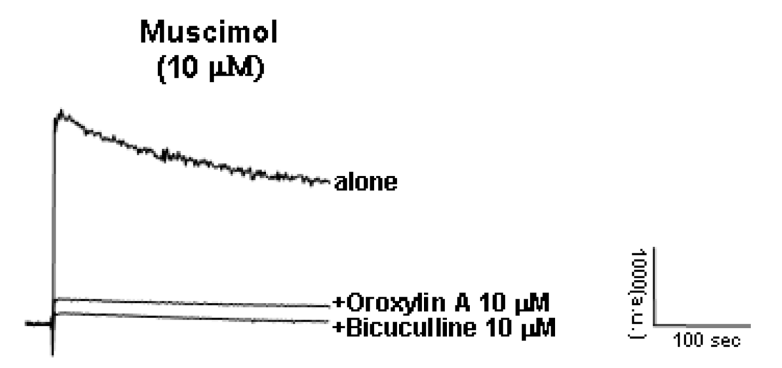

Fig. 5. Effects of oroxylin $\mathrm{A}$ on $\left[\mathrm{Cl}^{-}\right]_{\mathrm{i}}$ in neuroblastoma cells. Fluorescence was monitored at an excitation wavelength of $365 \mathrm{~nm}$ and an emission wavelength of $450 \mathrm{~nm}$ using the $\mathrm{Cl}^{-}-$ sensitive indicator, $N$-(6-methoxyquinolyl) acetoetylester (MQAE). Contents of influxed $\mathrm{Cl}^{-}$ion were expressed as a peak (a.u.). 
Flavonoids exhibit the different pharmacological spectra. For example, wogonin exhibited anxiolytic and anti-convulsive activities (Park et al., 2006; Park et al., 2007), while oroxyline A had memory enhancing effects (Kim et al., 2007; Kim et al., 2008). In the previous study, especially, oroxylin A exhibited an awakening effect in mice sleeping due to thiopental sodium administration, and the effect was similar to that of methylphenidate. This result suggested that oroxylin A might ameliorate ADHD behaviors.

In the current study, the behavioral characteristics of SHR and WKY were evaluated and compared using open field, elevated plus-maze, and rota-rod tests. SHR displayed hyperactivity, impulsivity and inattentiveness of motor coordination compared to WKY. These results are line with previous studies showing that SHR display hyperactivity, impulsivity, impaired ability to withhold responses, poorly sustained attention and reduced performance in different learning and memory paradigms (Prediger et al., 2005; Sagvolden et al., 2005; Sagvolden, 2000). Oroxylin A reduced hyperactivity in SHR (ADHD animal model) although it tended to increase locomotor activity in WKY (normal animal). But methylphenidate did not reduce hyperactivity. Behaviors such as rearing, moving to the central area and tendency to move into an unstable condition (open area in elevated plus-maze) were typically exhibited in impulsive animals (Pandolfo et., 2007; Ramos et al., 2002). Oroxylin A alleviated the impulsive behaviors such as rearing, the percentage of time moving to the central area and the tendency to move into an unstable condition (open area in elevated plus-maze). Methylphenidate also reduced percentage of staying time in the central area and the tendency to move into an unstable condition. The ability of the animal to stay on a rotating rod for long periods could represent sustained attention, a characteristic deficient in ADHD (Montgomery et al., 2008; Ferguson et al., 2003). Both oroxylin $A$ and methylphenidate enhance motor attention in SHR and WKY. These results indicate that oroxylin A may be a better choice for treatment of ADHD than methylphenidate because oroxylin A could alleviate all of hyperactivity, inattention and impulsivity while methylphenidate did not affect hyperactivity.

Many in vitro data indicate positive modulatory efficacies of Scutellaria flavones for the $\mathrm{GABA}_{\mathrm{A}}$ receptor via interaction with the benzodiazepine binding site(Michael et al., 2003; Hui et al., 2002). Michael et al. reported that oroxylin $\mathrm{A}$ inhibited $\left[{ }^{3} \mathrm{H}\right]$-flunitrazepam binding to rat cerebral cortical membrane and acted as an antagonist at the $\mathrm{GABA}_{\mathrm{A}}$ receptor benzodiazepine binding site (Michael et al., 2003).
GABA is the major inhibitory neurotransmitter in the CNS and is widely distributed in the neurons of the cortex. GABA contributes to motor control, vision and many other cortical functions. Some drugs that increase the level of GABA in the brain are used to treat epilepsy and to calm the trembling of patients suffering from Huntington's disease. The $\mathrm{GABA}_{\mathrm{A}}$ receptor is a member of the ligand-gated ion channel superfamily. Binding of GABA to the $\mathrm{GABA}_{\mathrm{A}}$ receptor activates chloride ion flux through the channel, and ligands for the benzodiazepine binding site modulate the inhibitory effects of GABA (Wang et al., 1999). GABAergic interneurons, which are the core component of cortico-limbic circuitry, are defective in the cerebral cortex of bipolar patients (Baar and Gntekin, 2008). GABA spreads in neural networks involved in cognitive and emotional processing and modulates noradrenergic, dopaminergic and serotonergic local neural circuitry (Brambilla et al., 2003). Low GABA activity is thought to be a genetically determined trait creating a vulnerability, that, in combination with environmental factors, can lead to the development of either mania or depression (Baar and Gntekin, 2008). It has also bee suggested that GABAergic dysfunction has direct or indirect pathogenetic importance in ADHD, and GABA receptor antagonists may become future pharmacotherapies for ADHD (Miyazaki et al., 2006; Weisler, 2007).

Benzodiazepine binding site ligands are classified as positive allosteric modulators, antagonists, or negative allosteric modulators according to their spectrum of intrinsic efficacy towards the $\mathrm{GABA}_{\mathrm{A}}$ receptor (Gardner et al., 1993). Positive allosteric modulators increase the frequency of chloride channel openings without altering the channel conductance or duration of opening. Therapeutically, they are used as anxiolytic, anti-convulsant, sedative-hypnotic, and muscle relaxant drugs. Many in vitro data indicate positive modulatory efficacies of wogonin and baicalein for the $G_{A B A}$ receptor via interaction with the benzodiazepine binding site and negative modulatory efficacy of oroxylin A (Michael et al., 2003; Hui et al., 2002). In this study, oroxylin $A$ inhibited the $\mathrm{Cl}^{-}$influx induced by muscimol, GABA receptor agonist. Therefore, the effects of oroxylin A may be caused by the antagonism at the $\mathrm{GABA}_{\mathrm{A}}$ receptor.

In summary, methylphenidate, a typical drug for ADHD treatment, significantly alleviated impulsivity (tendency to stay in central area and open arms) and inattentiveness of motor coordination (activity on rota-rod) in SHR but did not affect hyperactivity. Furthermore, it increased locomotor activities in WKY. In contrast, oroxylin A significantly alleviated hyperactivity, impulsivity (rearing frequency and 
tendency to stay in central area and open arms) and inattentiveness of motor coordination (activity on rota-rod) in SHR. The effect of oroxylin A may be caused by the antagonism at the $\mathrm{GABA}_{A}$ receptor. This result indicates that oroxylin $A$ may be a candidate drug for the treatment of ADHD.

\section{ACKNOWLEDGMENT}

This research was supported by a research grant from Sahmyook university.

\section{REFERENCES}

Basar, E. and Güntekin, B. (2008). A review of brain oscillations in cognitive disorders and the role of neurotransmitters. Brain Res. 1235, 172-193

Brambilla, P., Perez, J., Barale, F., Schettini, G and Soares, J. C. (2003). GABAergic dysfunction in mood disorders. Mol. Psychiatry 8, 721-737.

Brummelte, S., Grund, T., Moll, G. H., Teuchert-Noodt, G. and Dawirs, R. R. (2008). Environmental enrichment has no effect on the development of dopaminergic and GABAergic fibers during methylphenidate treatment of early traumatized gerbils. J. Negat. Results Biomed. 7, 2.

Dekermendjian, K., Kahnberg, P., Witt, M. R., Sterner, O., Nielsen, M., and Liljefors, T. (1999). Structure-activity relationships and molecular modeling analysis of flavonoids binding to the benzodiazepine site of the rat brain $\operatorname{GABA}(\mathrm{A})$ receptor complex. J. Med. Chem. 42, 4343-4350

Elia, J., Ambrosini, P. J. and Rapoport, J. L. (1999). Treatment of attention-deficit- hyperactivity disorder. N. Engl. J. Med. 340, 780-788.

Farkas, S., Berzsenyi, P., Kárpáti, E., Kocsis, P. and Tarnawa, I. (2005). Simple pharmacological test battery to assess efficacy and side effect profile of centrally acting muscle relaxant drugs. J. Pharmacol. Toxicol. Methods 52, 264-273.

Ferguson, S. A., Gray, E. P. and Cada, A. M. (2003). Early Behavioral Development in the Spontaneously Hypertensive Rat: A Comparison With the Wistar-Kyoto and SpragueDawley Strains. Behav. Neurosci. 117, 263-270.

Fone, K. C. and Nutt, D. J. (2005) Stimulants: use and abuse in the treatment of attention deficit hyperactivity disorder. Curr. Opin. Pharmacol. 5, 87-93.

Gardner, C. R., Tully, W. R. and Hedgecock, C. J. R. (1993). The rapidly expanding range of neuronal benzodiazepine receptor ligands. Prog. Neurobiol. 40, 1-61.

Hui, K. M., Michael, S. Y., Huen, H. Y. W., Zheng, H., Sigel, E., Baur, R., Ren, H., Li, Z. W., Wong, J. T. F. and Xue, H. (2002). Anxiolytic effect of wogonin, a benzodiazepine receptor ligand isolated from Scutellaria baicalensis Georgi. Biochem. Pharmacol. 64, 1415-1424.

Hui, K. M., Wang, X. H. and Xue, H. (2000). Interaction of flavones from the roots of Scutellaria baicalensis with the benzodiazepine site. Planta Med. 66, 91-93.

Jeong, J. O., An, N. Y., Park, S. H., Oh, J. G., Oh, H. L., Lee, B.
G. Om, A. S., Kim, B. S., Kim, D. H., Ryu, J. H. (2004). The Anxiolytic-like Effects of Scutellaria baicalensis Using Elevated Plus-Maze in Rats. Kor. J. Pharmacogn. 35, $22-27$.

Kim, D. H., Jeon, S. J., Son, K. H., Jung, J. W., Lee, S., Yoon, B. H., Choi, J. W. Cheong, J. H., Ko, K. H. and Ryu, J. H. (2006). Effect of the flavonoid, oroxylin A, on transient cerebral hypoperfusion-induced memory impairment in mice, Pharmacol. Biochem. Behav. 85, 658-668.

Kim, D. H., Jeon, S. J., Son, K. H., Jung, J. W., Lee, S., Yoon, B. H., Lee, J. J., Cho, Y. W., Cheong, J. H., Ko, K. H. and Ryu, J. H. (2007). The ameliorating effect of oroxylin A on scopolamine-induced memory impairment in mice, Neurobiol. Learning \& Memory 87, 536-546.

Kim, D. H., Kim, S., Jeon, S. J., Son, K. H., Lee, S., Yoon, B. H., Cheong, J. H., Ko, K. H. and Ryu, J. H. (2008). The effects of acute and repeated oroxylin A treatments on Abeta(25-35)induced memory impairment in mice. Neuropharmacol. 55, 639-647.

Kim, M. K., Yu, G. Y., Tan-Lee, B. S., Oh, H. J., Dong, K. W., Jeong, S. H., Han, S. W. and Cheong, J. H. (2003). Antistress effect of Pyroligneous liquid in SD rats and ICR mice. J. Appl. Pharmacol. 11, 249-256.

Kollins, S. H. MacDonald, E. K. and Rush, C. R. (2001). Assessing the abuse potential of methylphenidate in nonhuman and human subjects-a review. Pharmacol. Biochem. Behav. 68, 611-627.

Krause, K. H., Dresel, S. H., Krause, J., Fougere, C. and Ackenheil, M. (2003). The dopamine transporter and neuroimaging in attention deficit hyperactivity disorder. Neurosci. Biobehav. Rev. 27, 605-613.

Lee, G. S. Choi, J. Y., Ko, H. S., Tan-Lee, B. S., Yu, G. Y., Park, H. G. Kim, M. K., Ryu, J. H., Jung, I. K. and Cheong, J. H. (2006). Stress-reducing Effects of Brown Rice Koji. Food Sci. Biotech. 15, 63-69.

Lin, C. and Shieh, D. E. (1996). The anti-inflammatory activity of Scutellaria rivularis extracts and its active components, baicalin, baicalein and wogonin. Am. J. Chin. Med. 24, 31-36.

Michael S. Y. H., Justin W. C. L., Wah, Ng., Lui, W. S., Michelle, N. S. C., Wong, J. T. and Xue, H. (2003). 5,7-Dihydroxy-6methoxyflavone, a benzodiazepine site ligand isolated from Scutellaria baicalensis Georgi, with selective antagonistic properties. Biochem. Pharmacol. 66, 125-132.

Miyazaki, M., Ito, H., Saijo, T., Mori, K., Kagami, S. and Kuroda, Y. (2006). Favorable response of ADHD with giant SEP to extended-release valproate. Brain Develop. 28, 470-472.

Montgomery, K. S., Mackey, J., Thuett, K., Ginestra, S., Bizon, J. L. and Abbott, L. C. (2008). Chronic, low-dose prenatal exposure to methylmercury impairs motor and mnemonic function in adult C57/B6 mice. Behav. Brain Res. 191, 55-61.

Noldus, L. P. J. J., Spink, A. J. and Tegelenbosch, A. J. (2001). EthoVision: A versatile video tracking system for automation of behavioral experiments. Psychonomic Society 33, 398414.

Paladini, A. C., Marder, M., Viola, H., Wolfman, C., Wasowski, C. and Medina, J. H. (1999). Flavonoids and central nervous system: from forgotten factors to potent anxiolytic compounds. J. Pharm. Pharmacol. 51, 519-526.

Pandolfo, P., Pamplona, F. A., Prediger, R. D. S. and Taka- 
hashi, R. N. (2007). Increased sensitivity of adolescent spontaneously hypertensive rats, an animal model of attention deficit hyperactivity disorder, to the locomotor stimulation induced by the cannabinoid receptor agonist WIN 55,212-2. European J. Pharmacol. 563, 141-148.

Park, H. G., Choi, J. Y., Lee, G. S., Choi, J. H., Son, K. H., Ko, H. S., Ko, K. H., Ryu, J. H. and Cheong, J. H. (2006). Different effects of flavonoids in Scutellaria baicalensis on anxious and sedative behaviors. J. Appl. Pharmacol. 14, 83-89.

Park, H. G., Yoon, S. Y., Choi, J. Y., Lee, G. S., Shin, C. Y., Son, K. H., Lee, Y. S., Kim, W. K., Ryu, J. H., Ko, K. H. and Cheong J. H. (2007). Anticonvulsant effect of wogonin isolated from Scutellaria baicalensis. European J. Pharmacol. 574, 112-119.

Prediger, R. D., Pamplona, F. A., Fernandes, D. and Takahashi, R. N. (2005). Caffeine improves spatial learning deficits in an animal model of attention deficit hyperactivity disorder-the spontaneously hypertensive rat. Int. J. Neuropsychopharmacol. 8, 583-594.

Ramos, A., Kangerski, A.L. Basso, P.F., Da Silva, G.E. Santos, J.E., Assreuy, J., Vendruscolo, L.F. and Takahashi, R.N. (2002). Evaluation of Lewis and SHR rat strains as a genetic model for the study of anxiety and pain, Behav. Brain Res. 129, 113-123.

Sagvolden, T. (2000). Behavioral validation of the spontaneously hypertensive rat (SHR) as an animal model of attentiondeficit/hyperactivity disorder (AD/HD). Neurosci. Biobehav. Rev. 24, 31-39.

Sagvolden, T., Russell, V. A., Aase, H., Johansen, E. B. and Farshbaf, M. (2005). Rodent models of attention-deficit/hyper- activity disorder. Biol. Psychiatry 57, 1239-1247.

Salgueiro, J. B., Ardenghi, P., Dias, M., Ferreira, M. B. C., Izquierdo, I. and Medina, J. H. (1997). Anxiolytic natural and synthetic flavonoid ligands of the central benzodiazepine receptor have no effect on memory tasks in rat. Pharmacol. Biochem. Behav. 58, 887-891.

Shumaker, H., Amlal, H., Frizzell, R., Ulrich, C. D. Jr and Soleimani, M. (1999). CFTR drives $\mathrm{Na}^{+}-\mathrm{nHCO}^{-}$cotransport in pancreatic duct cells: a basis for defective $\mathrm{HCO}^{-}$secretion in CF. AM. J. Physiol. 276, C16-25.

Solanto, M. V. (2002). Dopamine dysfunction in AD/HD: integrating clinical and basic neuroscience research. Behav. Brain Res. 130, 65-71.

Tomimori, T., Miyaichi, Y. and Kizu, H. (1982). On the flavonoid constituents from the roots of Scutellaria baicalensis Georgi. Yakugaku Zasshi 102, 388-391.

Verkman, A. S., Sellers, M. C., Chao, A. C., Leung, T. and Ketcham, R. (1989). Synthesis and characterization of improved chloride-sensitive flurorescent indicators for biological applications. Anal. Biochem. 178, 355-361.

Wang, Q., Han, Y. and Xue, H. (1999). Ligands of GABAA receptor benzodiazepine binding site. CNS Drug Rev. 5, 125144.

Weisler, R. H. (2007). Emerging drugs for attention-deficit/hyperactivity disorder. Expert. Opin. Emerg. Drugs 12, 423-34.

West, M. R. and Molloy, C. R. (1996). A microplate assay measuring chloride ion channel activity. Anal. Biochem. 241, 5158. 